\title{
Inducible Nitric Oxide Distribution in the Fatty Liver of a Mouse with High Fat Diet-Induced Obesity
}

\author{
Seung-Kwon HA and Chanhee CHAE \\ Department of Veterinary Pathology, College of Veterinary Medicine, Seoul National University, \\ 56-1 San, Shillim-dong, Gwanak-gu, Seoul 151-742, Republic of Korea
}

\begin{abstract}
Obesity is a condition of abnormal adipose tissue storage and recently it has been recognized as a major factor in metabolic syndrome. High-fat diet-induced obesity in the C57BL/6 mouse is an important animal model because of its similarities with human obesity. The aim of the present study was to estimate obesity, liver injury and steatohepatitis, and the distribution of inducible nitric oxide synthase (iNOS) in mice with high-fat diet induced obesity. Three-week-old male C57BL/6J mice were fed either a high-fat diet (D-60: $60 \mathrm{kcal} \%$ fat, or D-45: $45 \mathrm{kcal} \%$ fat) or a normal diet (D-10: $10 \mathrm{kcal} \%$ fat) for 15 weeks. Oral glucose tolerance tests and intraperitoneal glucose tolerance tests showed that the D-60 mice had severely impaired glucose tolerance. In serum chemistry values and histopathological lesions, the D-60 group showed severe steatohepatitis. A distinct positive signal for iNOS was detected by immunohistochemistry in the cytoplasm of hepatocytes around the central vein in the D-45 and D-60 groups. Serum insulin levels and insulin immunohistochemistry in the pancreas showed pancreatic injury and insulin resistance in the D-60 group. We observed the presence of more iNOS in the high-fat diet-induced obese mouse, which has characteristics of nonalcoholic steatohepatitis (NASH) and diabetes, and expect that these background pathological data will be useful in research on obesity, diabetes mellitus, and non-alcoholic fatty liver disease.
\end{abstract}

Key words: C57BL/6 mouse, high fat diet, iNOS, non-alcoholic steatohepatitis, obesity

\section{Introduction}

Obesity is a condition of abnormal adipose tissue storage and recently it has been recognized as a major factor in metabolic syndrome, and is related to hypertension, hyperlipidemia, stroke, fatty liver, and diabetes mellitus $[15,26]$. Various animal models of obesity exist. For example, the $\operatorname{Lepr}^{d b}$ mouse, which is characterized by obesity, ketoacidosis, and hyperglycemia, and the $L e p^{d b}$, yellow, NZO mouse, which is characterized by obesity, hyperinsulinemia, and insulin resistance [35]. However, these models are based on monogenic disorders of little relevance to human obesity or diabetes. On the other hand, high-fat diet-induced obesity in the C57BL/6 mouse is an important animal model because of its similarities with human obesity $[9,35,40]$.

Non-alcoholic fatty liver disease (NAFLD) represents a spectrum of liver diseases encompassing steatosis (fatty change), non-alcoholic steatohepatitis (NASH), and cirrhosis in the absence of alcohol abuse [3]. Recent

(Received 7 February 2010 / Accepted 2 May 2010)

Address corresponding: C. Chae, Department of Veterinary Pathology, College of Veterinary Medicine, Seoul National University, 56-1 San, Shillim-Dong, Gwanak-Gu, Seoul 151-742, Republic of Korea 
studies have shown that NAFLD is strongly associated with obesity, dyslipidemia, and type II diabetes mellitus [31-33]. A diagnosis of NASH is made after findings of combination of steatosis, hepatocyte injury and necrosis, mixed inflammatory infiltration and variable degrees of fibrosis shown histopathologically [25]. The pathogenesis of NASH remains unclear but it has recently been suggested that excessive intrahepatic lipid accumulation may trigger local inflammatory responses $[1,3,23]$.

Nitric oxide (NO) is the enzymatic product of a group of hemoproteins collectively known as NO synthase (NOS) that catalyze the oxidation of one of the guanidino groups of L-arginine, giving rise to equimolar amounts of citrulline and NO. Of the three isoforms of NOS, inducible NO synthase (iNOS), also designated NOS2 is the most strongly associated with antimicrobial activity [7]. Also, iNOS is assumed to be one of the mediators of inflammation-involved insulin resistance [12]. From a functional perspective, it is important to recognize the induction and distribution of iNOS in steatohepatitis. However, whether iNOS is expressed in livers with steatohepatitis is still unclear. The objective of this study was, therefore, to investigate immunohistochemically the presence and distribution of iNOS in the tissues of mouse livers with high fat-induced steatohepatitis, and throw further light on the pathogenesis of NASH to better understand the relationship between steatosis, inflammation, and insulin resistance.

\section{Materials and Methods}

\section{Animals}

A total of 45 male 3-week-old C57BL/6J mice were purchased from Orient Bio Inc. (Gyeonggi-Do, Korea). One week after arrival, the mice were divided into three groups based on diet. The mice were fed either a highfat diet (D-60 group: $60 \mathrm{kcal} \%$ fat, D12492, Research Diet Inc., New Brunswick, NJ, USA; or D-45 group: 45 kcal\% fat, D12451, Research Diet Inc.) or a normal diet (D-10 group: 10 kcal\% fat, D12450B, Research Diet Inc.) for 18 weeks. All mice were housed in polycarbonate cages in a room maintained at $21 \pm 2^{\circ} \mathrm{C}$ with a relative humidity of $50 \pm 5 \%$. Ventilation was set at $10-20$ changes per hour. The room was lighted automatically from 07:00 $\mathrm{h}$ to 19:00 $\mathrm{h}$. The animals were allowed free access to reverse-osmosis filtered, UV-treated, chlorinated tap water. Noise and ammonia were below $60 \mathrm{~dB}$ and $20 \mathrm{ppm}$, respectively. The experiments were conducted in accordance with the Institutional Animal Care and Use Committee (IACUC) of Seoul National University.

\section{Glucose tolerance tests}

After 15 weeks, when the animals were 18 weeks of age, we performed an oral glucose tolerance test (OGTT) and intraperitoneal glucose tolerance test (IPGTT). The mice were fasted for $16 \mathrm{~h}$, and their body weights were measured for the conversion glucose dose. Glucose dosing was performed using a $1 \mathrm{ml}$ syringe and feeding needle. Blood was drawn from the tail vein and the blood glucose was measured using a glucose meter (AccuCHEK Sensor, Roche Diagnostic Corp., Indianapolis, IN, USA) at 0, 30, 60, 90, and 120 min after the oral and i.p. injection of glucose $(2 \mathrm{~g} / \mathrm{kg}$ body weight). In the IPGTT, glucose was also measured at $150 \mathrm{~min}$.

\section{Organ weight, serum chemistry}

After 15 weeks, the animals were sacrificed with an overdose of $\mathrm{CO}_{2}$. Blood was collected from the heart, and centrifuged at $3,000 \mathrm{rpm}$ for $15 \mathrm{~min}$ at $4^{\circ} \mathrm{C}$. Serum chemistry for aspartate aminotransferase (AST), alanine aminotransferase (ALT), total cholesterol, triglycerides (TG), high density lipoprotein (HDL-C), low density lipoprotein (LDL-C), phospholipids and free fatty acid (NEFA) was analyzed by an automatic chemistry analyzer (Hitachi 7180, Hitachi Co., Ltd., Tokyo, Japan). In the analysis of AST, ALT, total cholesterol, phospholipids and NEFA, Wako reagents (Wako Pure Chemical Industries, Ltd., Osaka, Japan) were used, and for the HDL-C and LDL-C, Sekisui reagents were used (Sekisui Chemical Co., Ltd., Osaka, Japan). The liver weight, and fat pad weight (sum of epididymal fat and retroperitoneal fat) were also measured.

\section{Serum insulin level}

Insulin levels in the mouse serum were assessed in duplicate using an ELISA kit (Mouse Insulin ELISA TMB Kit; Shibayagi Co., Ltd., Gunma, Japan). The insulin level in the serum was determined according to 
the manufacturer's instructions. A standard curve was prepared ranging between 0.125 and $3 \mathrm{ng} / \mathrm{ml}$. The insulin concentration was calculated from this standard curve.

\section{Liver histology}

Livers were fixed in $10 \%$ buffered formalin and embedded in paraffin, cut at a thickness of $5 \mu \mathrm{m}$ on a microtome, placed on slides and stained with hematoxylin and eosin. Each section was then scored for inflammation and fibrosis separately by a Board-certified toxicologic pathologist according to the published criteria [7, 25,28 ]. For steatosis: up to $25 \%$ steatosis (score 1); 26 to $50 \%$ steatosis (score 2); 51 to $75 \%$ (score 3); more than $76 \%$ steatosis (score 4). For inflammatory grade: focal collection of mononuclear cells (score 1); diffuse infiltrates of mononuclear cells (score 2); focal collection of polymorphonuclear cells (score 3); diffuse infiltrates of polymorphonuclear cell (score 4).

\section{iNOS immunohistochemistry in the liver}

Liver tissues were sectioned at $5 \mu \mathrm{m}$, and mounted on poly-L-lysine coated slides. The sections were routinely dewaxed and rehydrated. Endogenous peroxidase was quenched with $3 \%$ hydrogen peroxide for $10 \mathrm{~min}$ at room temperature. Slides were washed with distilled water for $5 \mathrm{~min}$ and placed in plastic Coplin jars containing sodium citrate buffer ( $\mathrm{pH}$ 6.0). The jars were covered with screw caps and heated in a domestic microwave oven at the highest setting $(800 \mathrm{~W})$ for $30 \mathrm{~min}$. The heating time was divided into three cycles with an interval of $5 \mathrm{~min}$ between cycles to check on the fluid level in the jars. After heating, the Coplin jars were removed from the oven and allowed to cool for $15 \mathrm{~min}$. Rabbit polyclonal iNOS antibody (AB Cam, Cambridge, MA, USA) was diluted 1:200 in antibody diluents (Dako, Glostrup, Denmark). The slides were incubated with antibody overnight at $4{ }^{\circ} \mathrm{C}$ in a humid chamber. After three washes with $0.1 \%$ Tween 20 in Tris buffer ( $\mathrm{pH} 7.4$ ), the sections were flooded with dextran polymer (Envision kit, Dako) and incubated for $30 \mathrm{~min}$ at room temperature. The sections were then washed three times with Tris buffer ( $\mathrm{pH}$ 7.4). The final reaction was produced by immersing the sections in a solution of 3,3'-diaminobenzidine (DAB) for $1 \mathrm{~min}$ at room temperature.
The sections were lightly counterstained with Real Hematoxylin (Dako) and blued in $0.2 \%$ ammonia water.

\section{Morphometric image analysis}

For morphometric analysis of the iNOS, three fields from each liver were captured by digital camera (TDI Digicam HQ, Seoul, Korea). Image J software (National Institutes of Health, Bethesda, MD, USA) was used for computerized quantification of the immunostained area. Automatic detection of DAB reactions was performed using a colorimetric approach. DAB positive pixels were selectively detected using the "threshold color function." For the intensity of the DAB positive pixel, gray density (0: white; 255 : black) was also measured for the whole image.

\section{Insulin immunohistochemistry in the pancreas}

The immunohistochemistry method used for the pancreas was similar to the iNOS immunohistochemistry described above. The primary antibody was guinea pig anti-insulin antibody (Dako), and antigen retrieval was not performed.

\section{Statistical analysis}

Results are presented as the mean and SD. Data were analyzed by one-way analysis of variance (ANOVA) incorporating Bartlett's test for homogeneity of variance. Where variances were found to be homogenous, pairwise comparisons were conducted using Dunnett's test. Where Bartlett's test found unequal variances among group data, the affected parameters were analyzed using non-parametric Kruskal-Wallis and Dunn's test. The semi-quantitative liver score was also analyzed using a non-parametric test. GraphPad Prism ${ }^{\circledR}$ Version 4.03 (GraphPad Software, San Diego, CA, USA) was used for all statistical analyses.

\section{Results}

\section{Body weight and organ weight}

The body weight curve is shown in Table 1 and Fig. 1. At 18 weeks of age ( 15 weeks into the study period), the D-10 group had a mean weight of $29.75 \pm 2.01 \mathrm{~g}$, the D-45 group $35.30 \pm 2.49 \mathrm{~g}(P<0.001$ compared to D-10), and the D-60 group $44.59 \pm 1.55 \mathrm{~g}(P<0.001$ compared 
Table 1. Terminal body weight, and liver and fat pad weights of C57BL/6J mice fed high-fat (D-45, D-60) and normal (D-10) diets

\begin{tabular}{lccc}
\hline & D-10 group & D-45 group & D-60 group \\
\hline Terminal body weight $(\mathrm{g})$ & $29.75 \pm 2.01$ & $35.30 \pm 2.49^{* *}$ & $44.59 \pm 1.55^{* *}$ \\
Absolute liver weight $(\mathrm{g})$ & $0.97 \pm 0.11$ & $1.03 \pm 0.13$ & $1.63 \pm 0.24^{* * *}$ \\
Relative liver weight $(\%$ of terminal body weight) & $3.27 \pm 0.27$ & $2.90 \pm 0.19^{*}$ & $3.66 \pm 0.46$ \\
Absolute fat pad weight $(\mathrm{g})$ & $2.28 \pm 0.58$ & $3.77 \pm 0.64^{* *}$ & $4.02 \pm 0.83^{* *}$ \\
Relative fat pad weight $(\%$ of terminal body weight) & $7.57 \pm 1.57$ & $10.65 \pm 1.51^{* *}$ & $9.06 \pm 2.03$ \\
\hline
\end{tabular}

$* P<0.05, * * P<0.01, * * * P<0.001$ compared to the $\mathrm{D}-10$ group.

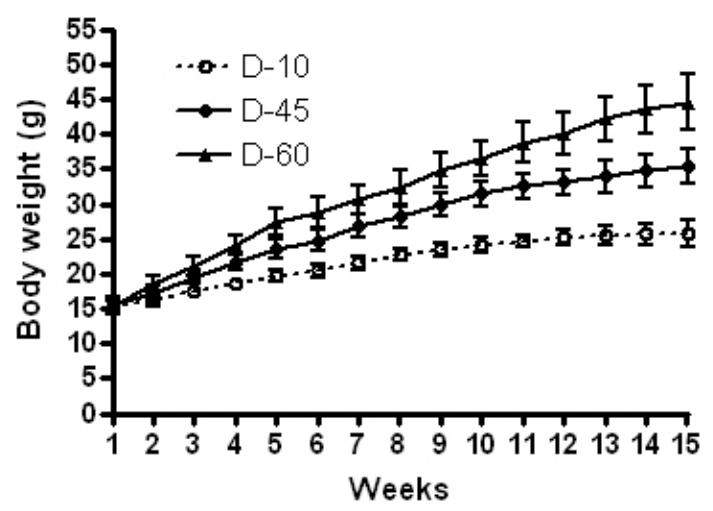

Fig. 1. Body weight changes of C57BL/6J mice fed normal (D-10) or high-fat (D-45, D-60) diets for 15 weeks. Data represent mean \pm SD of 15 mice per group.

to D-10). During the 15-week study period, the D-10 mice gained approximately $12.45 \mathrm{~g}$ of body weight, the D-45 mice gained $19.6 \mathrm{~g}$, and the D-60 mice gained $29.19 \mathrm{~g}$.

In absolute organ weight, the liver and fat pad weighed more in the D-60 and D-45 groups than the D-10 group $(P<0.05)$ (Table 1$)$.

\section{Glucose tolerance tests}

Figure 2 shows the OGTT and IPGTT results obtained in this study. The blood glucose level in all mice increased for $30 \mathrm{~min}$ after oral or intraperitoneal glucose administration and then declined to basal values. For both tests, the glucose level of the D-60 group was significantly higher than that of the D-10 group before the dose and 30, 60, 90, and $120 \mathrm{~min}$ after the dose $(P<0.01)$. Also in both tests, the impaired glucose tolerance was more severe in the D-60 group than in the D-45 group. In the IPGTT, the glucose level of the D-45 group was also significantly higher than that of the D-10 group before the dose and 30,60, 90, and $120 \mathrm{~min}$ after the dose $(P<0.05)$.

\section{Serum chemistry and insulin level}

We found that the serum ALT level was increased 4 -fold in the D-60 group $(P<0.001)$ compared to the D-45 and D-10 groups. AST, total cholesterol, TG and LDL-C, NEFA and phospholipid levels were also higher in the D-60 group $(P<0.05)$ than in the D-45 and D-10 groups, but HDL-C was not significantly different among the groups and LDL-C showed no correlation with diet fat contents (Table 2).

ELISA of serum insulin levels indicated that the insulin level was $0.76 \pm 0.27 \mathrm{ng} / \mathrm{ml}$ in the D-10 group, 1.30 $\pm 0.55 \mathrm{ng} / \mathrm{ml}$ in the D-45 group and $2.54 \pm 0.99 \mathrm{ng} / \mathrm{ml}$ in the D-60 group. Serum insulin was present at significantly higher levels $(P<0.01)$ in the $\mathrm{D}-60$ group than in the D-10 group (Table 2).

\section{Histopathology}

Severe centrilobular to diffuse fatty change (steatosis) in the liver was observed in the high-fat diet groups. At higher magnification, mixed cytoplasmic microvacuolation and macrovacuolation were observed in hepatocytes from the D-60 and D-45 groups. Microvacuole infiltration in the hepatocytes was more severe in the D-60 group than in the D-45 group. In addition, inflammatory cell infiltration was observed in the D-45 group and the D-60 group. Inflammatory cells mainly consist of polymorphonuclear neutrophils and mononuclear cells. Sometimes focal necrosis, perisinusoidal and portal fibrosis, and severe hepatocellullar ballooning were observed in the D-45 and D-60 groups. In the semi-quantitative analyses of steatosis and inflammatory grade, the 

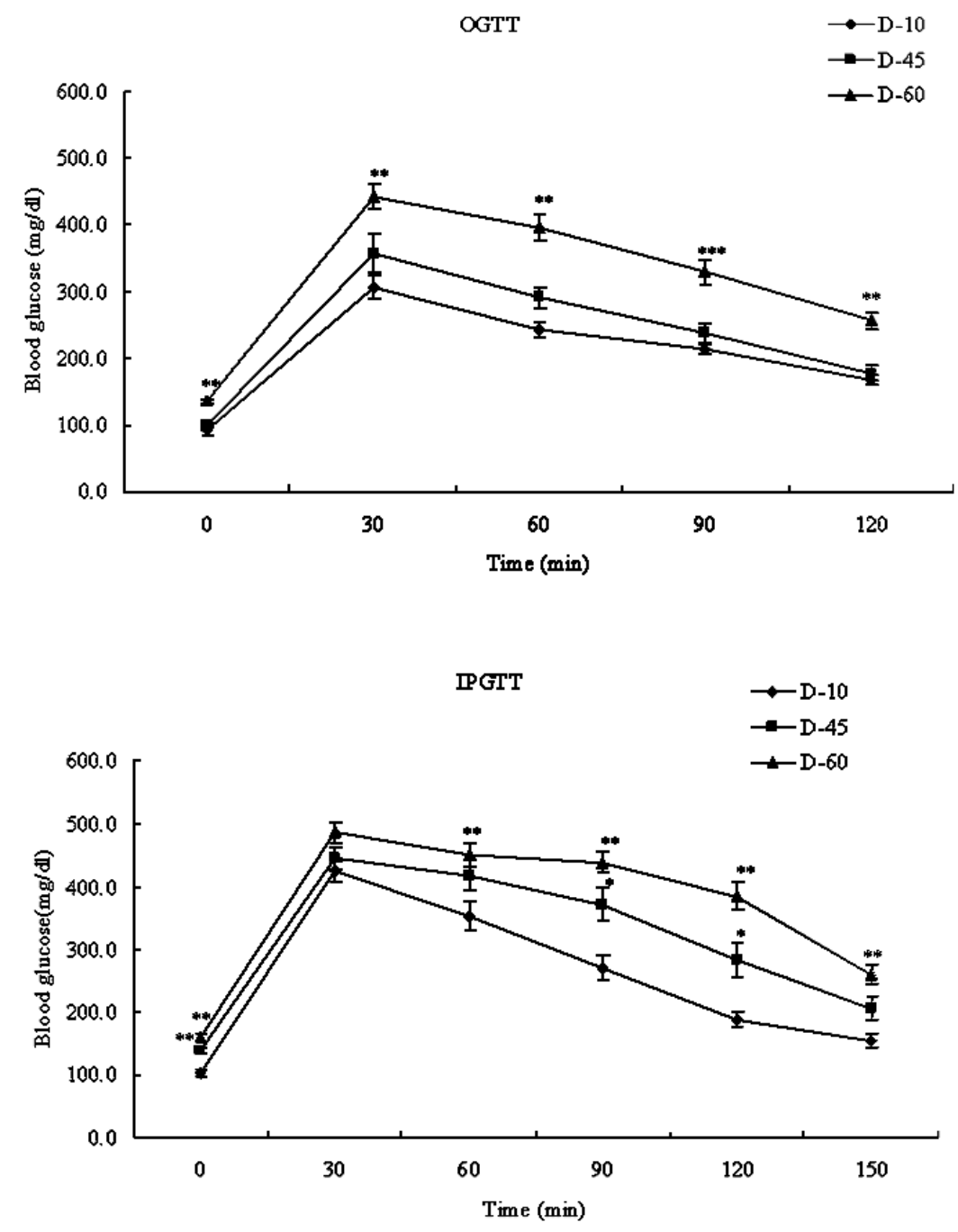

Fig. 2. Oral glucose tolerance test (OGTT) and intraperitoneal glucose tolerance test (IPTGG) result for C57BL/6J mice fed high-fat (D-45, D-60) or normal (D-10) diets for 15 weeks prior to the tests. Time 0 indicates the point when the rats were given the conversion glucose dose orally or intraperitoneally. Data represent mean \pm SE of 15 mice per group; $* * P<0.01$, $* * * P<0.001$.

Table 2. Serum chemistry values and insulin level in C57BL/6J mice fed high-fat (D-45, D-60) and normal (D-10) diets

\begin{tabular}{lccc}
\hline & D-10 group & D-45 group & D-60 group \\
\hline AST (IU/l) & $112.18 \pm 71.86$ & $125.04 \pm 79.50$ & $184.74 \pm 104.57 *$ \\
ALT (IU/l) & $59.78 \pm 26.12$ & $69.96 \pm 32.13$ & $201.49 \pm 52.67 * * *$ \\
Total cholesterol (mg/dl) & $119.4 \pm 15.91$ & $118.8 \pm 16.84$ & $164.1 \pm 25.68 *$ \\
Triglycerides $(\mathrm{mg} / \mathrm{dl})$ & $47.33 \pm 13.22$ & $58.20 \pm 21.64$ & $63.93 \pm 20.97 *$ \\
HDL cholesterol $(\mathrm{mg} / \mathrm{dl})$ & $61.40 \pm 7.52$ & $60.50 \pm 8.76$ & $66.04 \pm 6.35$ \\
LDL cholesterol $(\mathrm{mg} / \mathrm{dl})$ & $10.13 \pm 2.48$ & $8.40 \pm 3.17$ & $13.85 \pm 3.85^{* *}$ \\
Phospholipids $(\mathrm{mg} / \mathrm{dl})$ & $190.3 \pm 24.44$ & $191.2 \pm 28.79$ & $233.5 \pm 30.31^{* *}$ \\
NEFA $(\mathrm{mEq} / \mathrm{l})$ & $0.94 \pm 0.10$ & $1.14 \pm 0.35$ & $1.29 \pm 0.21 * *$ \\
Insulin $(\mathrm{ng} / \mathrm{ml})$ & $0.76 \pm 0.27$ & $1.30 \pm 0.55$ & $2.54 \pm 0.99 * *$ \\
\hline
\end{tabular}

$* P<0.05, * * P<0.01, * * * P<0.001$ compared to the $\mathrm{D}-10$ group. 


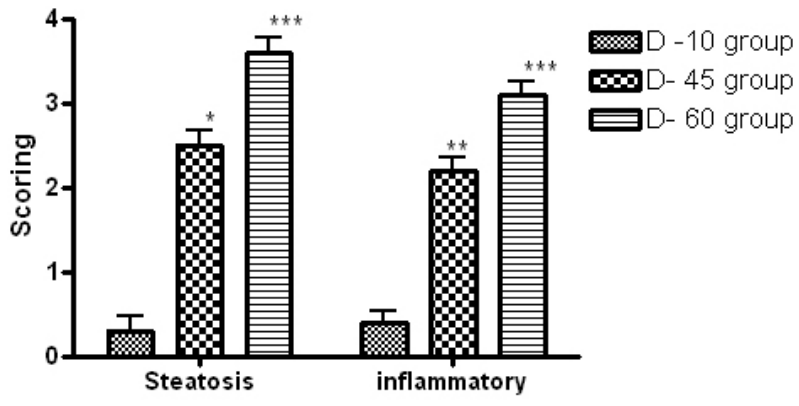

Fig. 3. Semi-quantitative histopathological analysis of C57BL/6J mice fed high-fat (D-45, D-60) or normal (D-10) diets, Data represent mean \pm SE of 15 mice per group; $* P<0.05$, $* * P<0.01, * * * P<0.001$.

scores of the D-45 and D-60 groups were significantly higher $(P<0.01)$ than those of the D-10 group (Fig. 3).

\section{iNOS immunohistochemistry}

A distinct positive signal for iNOS was detected in the cytoplasm of hepatocytes around the central vein in the D-45 and D-60 groups (Fig. 4). The signal was observed primarily in the area of vacuolation. The signal was stronger in the vacuolated hepatocytes and macrophages than in the normal hepatocytes. iNOS was also detected in hepatocytes from the D-10 group, but to a lesser extent than in the other groups. In the morphometric image analyses, the iNOS stained area of the D-10 group was $67,884 \pm 24,340 \mu \mathrm{m}^{2}$, that of the D-45 group was $103,705 \pm 25,466 \mu \mathrm{m}^{2}(P<0.01$ vs. D-10), and that of the D-60 group was $115,754 \pm 20,762 \mu \mathrm{m}^{2}(P<0.01$ vs. D-10). The gray density of the D-10 group was 136.2 \pm 7.3 , that of the D-45 group was $161.0 \pm 7.2(P<0.01)$ and that of the D-60 group was $167.5 \pm 9.914(P<0.001$ vs. D-10). The iNOS areas and densities were significantly larger in the high-fat diet groups (D-45 and D-60 groups) than in the $\mathrm{D}-10$ group.

\section{Insulin immunohistochemistry in the pancreas}

We performed insulin immunohistochemistry in the pancreas and evaluated beta cell and endocrine injury. We found that 11 of 15 pancreata from the D-60 group and 4 of 15 pancreata from the D-45 group were injured. These injured pancreata had increasingly irregular islet boundaries, leading to the appearance of endocrine cell projections into the surrounding exocrine tissue, and
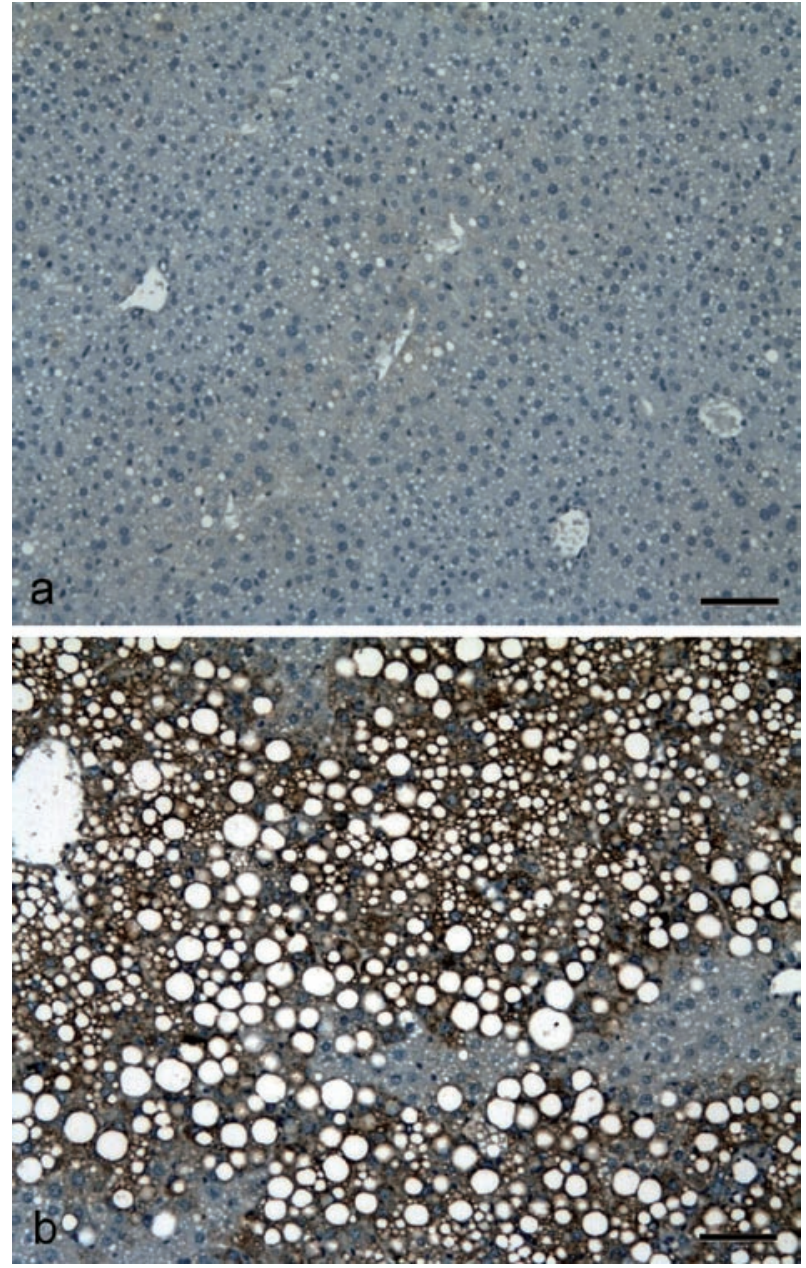

Fig. 4. Immunohistochemistry for iNOS using DAB and hematoxylin counterstain. a, D-10 group; b, D-60 group. Scale bar $=50 \mu \mathrm{m}$.

insulin staining was reduced (Fig. 5). Injured islets were not observed in the D-10 group.

\section{Discussion}

In this study, we evaluated the baseline pathological data of mice fed a high-fat diet as a model of obesity, NASH and type 2 diabetes, and immunohistochemically investigated the presence and distribution of iNOS in the tissues of mouse livers with high fat-induced steatohepatitis.

In the OGTT and IPGTT, we confirmed impaired glucose tolerance in the high fat groups, particularly the D-60 group. The D-60 group also had increased termi- 



Fig. 5. Immunohistochemistry for insulin in the pancreas using DAB and hematoxylin counterstain. a, D-10 group; b, D-60 group. Scale bar $=50 \mu \mathrm{m}$.

nal body weight and liver and fat pad weights compared to the D-10 group.

ALT is a leakage enzyme, reflecting cell membrane function, with the highest concentrations in mice found in the liver. AST is a leakage enzyme associated with both the cytosol and mitochondria in mice. Despite their widespread tissue distribution, ALT and AST are useful markers for measuring hepatocellular damage [21]. We found that both AST and ALT were increased in the D-60 group. Thus, we assume that the high-fat diet induced liver damage, increasing the AST and ALT levels and histopathologcal lesions.

In this study, TG and total cholesterol, phospholipids and NEFA were increased in the D-60 group, but HDL-
C was not significantly changed, and LDL-C showed no observed in correlation with diet fat contents. In general, obesity, atherosclerosis or lipidemia has been shown to be associated with increased total cholesterol and LDL-C, and decreased HDL-C in human. Total cholesterol in different strains of mice ranges between 55-128 $\mathrm{mg} / \mathrm{dl}$, one quarter to one half that in human beings [17]. In mice, most of the total cholesterol (80-90\%) of mice fed with rodent chow diet is found in HDL-C, and the small fraction not found in HDL is mostly VLDL [29]. Mice also lack an equivalent of human CETP (cholesterol ester transfer protein) [29]. Accordingly, some researchers argue that HDL-C and LDL-C are unsuitable biomarkers of lipid metabolism, and should be interpreted with care HDL-C or LDL-C measurements in mouse models of hyperlipidemia $[4,11,21]$. Serum phospholipids mirror the dietary intake of recent months and also roughly mirror the composition of the body fat [18]. Serum phospholipids and NEFA are thought to be key initiating factors in NASH pathogenesis that results in increased generation of reactive oxygen species leading to damage of biological membranes [44]. Thus, we suggest that serum phospholipids and NEFA may be markers of the obesity and NASH status.

In this study, inflammatory cell infiltration was observed in the high-fat diet groups. The overall inflammatory cell distribution shifted toward polymorphonuclear neutrophils as steatosis progressed. Also, the induction of iNOS in the liver seemed to correlate with fatty changes. The expression of iNOS is up-regulated by most inducers of obesity, hyperglycemia, and insulin resistance, including free fatty acids, endotoxins, and oxidative stress [12]. We observed a strong iNOS signal in the livers from mice fed a high-fat diet. Serum chemistry, histopathology, and iNOS distribution, in the highfat diet-induced obesity mouse seemed to indicate steatohepatitis, and iNOS was more abundant in this model.

The pathogenesis of NASH is not well established, but many studies have found that insulin resistance is a primary mediator of hepatic steatosis, the 'first hit' for the disease. The 'second hit' is steatosis sensitizing the liver to inflammation, oxidative stress, mitochondrial dysfunction, and fibrosis [1, 3, 24]. The association between obesity and NAFLD has prompted studies on 
the effects of diet on the development of steatosis and steatohepatitis in mouse models. An increased dietary supply of fat or carbohydrates to the liver may promote steatosis by increasing hepatic lipid uptake. In this pathway, oxidative stress appears to play a key role in the pathogenesis of NASH [10, 20], and nitric oxide may potentiate cytotoxicity through its reaction with superoxide anion and formation of nitrotyrosine $[13,14]$. The finding that an abnormal intrahepatic accumulation of nitrotyrosine is associated with the histological severity of NASH strongly suggests that NO-related oxidative injury may play a significant role in the pathogenesis of this liver disease [14]. Some studies have suggested that obese mice with severe steatosis show that liver injury is mediated by both TNF- $\alpha$ and IFN- $\gamma[42,43]$. Since it has been demonstrated that proinflammatory cytokines are directly involved in the up-regulation of iNOS [22, 37], we speculate that locally-released proinflammatory molecules could play a significant role in the pathogenesis of NASH.

In this study, the serum insulin level was increased, and pancreatic beta cells were injured in the high-fat diet group. Reduced pancreatic beta cell mass and beta cell dysfunction are major factors in the pathogenesis of type 2 diabetes. Decreased beta cell proliferation and cellular senescence are found in mice with diet-induced type 2 diabetes [36]. We observed pancreatic beta cell injury and insulin resistance in the high-fat diet group in this study, too. Because steatosis and insulin resistance can cause or potentiate each other, it remains unclear as to whether steatosis or insulin resistance is the initiating step of NAFLD. Recently, it has been shown that steatosis may arise from causes unrelated to diet and then gives rise to hepatic insulin resistance [19, 30, 34]. Another study has shown that steatosis is associated with activation of NF- $\kappa \mathrm{B}$ and this pathogenic role of inflammation is meaningful in the development of hepatic insulin resistance $[5,14]$. NF- $\mathrm{-B}$ is one of the most important nuclear proteins involved in the cytokine-induced transcriptional induction of the iNOS gene in various cell types including macrophages $[5,28]$. Further study is needed to elucidate whether hepatic insulin resistance is primary or secondary to steatosis, and how iNOS interact to expand hepatic lipid stores, cause hepatocellular injury and recruit inflammation.
The C57BL/6 mouse given a high-fat diet is considered a good model for investigating metabolic and environmental NASH $[8,41]$. This model was initially studied by Surwit et al. in 1988 [38]. C57BL/6 mice are genetically predisposed to obesity, hyperinsulinemia, and glucose intolerance with age irrespective of diet. This phenotype can be promoted and histopathologically mixed micro- and macrovacuolation (steatosis) has been observed after feeding the mice a high-fat diet over a long period of time. The reason for this seems to be spontaneous mutation in the nicotinamide nucleotide transhydrogenase gene carried by C57BL/ 6 mice, which induces glucose intolerance [39]. C57BL/6 mice are a useful diet-induced obesity and type II diabetes model compared to other strains $[2,16,40]$.

In conclusion, we studied steatohepatitis in mice with high-fat diet-induced obesity and investigated whether the high-fat diet-induced obesity mouse is a good model for NASH. Our present data show that iNOS is more highly expressed in high-fat-diet induced steatoheapatits. We expect that these data will be useful for research into obesity, diabetes mellitus, and non-alcoholic fatty liver disease.

\section{References}

1. Adams, L.A., Angulo, P., and Lindor, K.D. 2005. Nonalcoholic fatty liver disease. CMAJ 172: 899-905.

2. Ahren, B. and Scheurink, A.J. 1998. Marked hyperleptinemia after high-fat diet associated with severe glucose intolerance in mice. Eur. J. Endocrinol. 139: 461-467.

3. Anstee, Q.M. and Goldin, R.D. 2006. Mouse models in non-alcoholic fatty liver disease and steatohepatitis research. Int. J. Exp. Pathol. 87: 1-16.

4. Breslow, J. 1996. Mouse models of atherosclerosis. Science 272: 685 .

5. Cai, D., Yuan, M., Frantz, D.F., Melendez, P.A., Hansen, L., Lee, J., and Shoelson, S.E. 2005. Local and systemic insulin resistance resulting from hepatic activation of IKK-beta and NF-kappaB. Nat. Med. 11: 183-190.

6. Chen, S., Chen, Y., Shi, J., Lin, Y., and Xie, W. 2006. The restorative effect of taurine on experimental nonalcoholic steatohepatitis. Dig. Dis. Sci. 51: 2225-2234.

7. Cho, W.S. and Chae, C. 2002. Immunohistochemical detection and distribution of inducible nitric oxide synthase in pigs naturally infected with Actinobacillus pleuropneumoniae. J. Comp. Pathol. 126: 109-114.

8. Collins, S., Martin, T.L., Surwit, R.S., and Robidoux, J. 2004. Genetic vulnerability to diet-induced obesity in the C57BL/6J mouse: physiological and molecular characteristics. Physiol. Behav. 81: 243-248. 
9. Comuzzie, A.G. and Allison, D.B. 1998. The search for human obesity genes. Science 280: 1374-1377.

10. Day, C. and James, O. 1998. Hepatic steatosis: innocent bystander or guilty party? Hepatology (Baltimore, Md.) 27: 1463.

11. Escola-Gil, J.C., Jorba, O., Julve-Gil, J., Gonzalez-Sastre, F., Ordonez-Llanos, J., and Blanco-Vaca, F. 1999. Pitfalls of direct HDL-cholesterol measurements in mouse models of hyperlipidemia and atherosclerosis. Clin. Chem. 45: 1567-1569.

12. Fujimoto, M., Shimizu, N., Kunii, K., Martyn, J.A., Ueki, K., and Kaneki, M. 2005. A role for iNOS in fasting hyperglycemia and impaired insulin signaling in the liver of obese diabetic mice. Diabetes 54: 1340-1348.

13. Garcia-Monzon, C., Majano, P.L., Zubia, I., Sanz, P., Apolinario, A., and Moreno-Otero, R. 2000. Intrahepatic accumulation of nitrotyrosine in chronic viral hepatitis is associated with histological severity of liver disease. $J$. Hepatol. 32: 331-338.

14. Garcia-Monzon, C., Martin-Perez, E., Iacono, O., FernandezBermejo, M., Majano, P., Apolinario, A., Larranaga, E., and Moreno-Otero, R. 2000. Characterization of pathogenic and prognostic factors of nonalcoholic steatohepatitis associated with obesity. J. Hepatol. 33: 716-724.

15. James, P.T., Leach, R., Kalamara, E., and Shayeghi, M. 2001. The worldwide obesity epidemic. Obes. Res. 9: 228S-233S.

16. Jiang, T., Wang, Z., Proctor, G., Moskowitz, S., Liebman, S.E., Rogers, T., Lucia, M.S., Li, J., and Levi, M. 2005. Diet-induced obesity in C57BL/6J mice causes increased renal lipid accumulation and glomerulosclerosis via a sterol regulatory element-binding protein-1c-dependent pathway. J. Biol. Chem. 280: 32317-32325.

17. Jiao, S., Cole, T., Kitchens, R., Pfleger, B., and Schonfeld, G. 1990. Genetic heterogeneity of plasma lipoproteins in the mouse: control of low density lipoprotein particle sizes by genetic factors. J. Lipid Res. 31: 467.

18. Karlsson, M., Marild, S., Brandberg, J., Lonn, L., Friberg, P., and Strandvik, B. 2006. Serum phospholipids fatty acids, adipose tissue, and metabolic markers in obese adolescents\&ast. Obesity 14: 1931-1939.

19. Larter, C.Z. and Farrell, G.C. 2006. Insulin resistance, adiponectin, cytokines in NASH: Which is the best target to treat? J. Hepatol. 44: 253-261.

20. Letteron, P., Fromenty, B., Terris, B., Degott, C., and Pessayre, D. 1996. Acute and chronic hepatic steatosis lead to in vivo lipid peroxidation in mice. J. Hepatol. 24: 200208.

21. Loeb, W.F. and Quimby, F.W. 1989. The Clinical Chemistry of Laboratory Animals, Pergamon, New York.

22. Majano, P.L., Garcia-Monzon, C., Lopez-Cabrera, M., LaraPezzi, E., Fernandez-Ruiz, E., Garcia-Iglesias, C., Borque, M.J., and Moreno-Otero, R. 1998. Inducible nitric oxide synthase expression in chronic viral hepatitis. Evidence for a virus-induced gene upregulation. J. Clin. Invest. 101: 1343-1352.

23. Marchesini, G., Bugianesi, E., Forlani, G., Cerrelli, F., Lenzi, M., Manini, R., Natale, S., Vanni, E., Villanova, N., Melchionda, N., and Rizzetto, M. 2003. Nonalcoholic fatty liver, steatohepatitis, and the metabolic syndrome. Hepatology 37: 917-923.

24. Matteoni, C., Younossi, Z., Gramlich, T., Boparai, N., Liu, Y., and McCullough, A. 1999. Nonalcoholic fatty liver disease: a spectrum of clinical and pathological severity. Gastroenterology 116: 1413-1419.

25. McCullough, A. 2004. The clinical features, diagnosis and natural history of nonalcoholic fatty liver disease. Clin. Liver Dis. 8: 521-533.

26. Mokdad, A.H., Ford, E.S., Bowman, B.A., Dietz, W.H., Vinicor, F., Bales, V.S., and Marks, J.S. 2003. Prevalence of obesity, diabetes, and obesity-related health risk factors, 2001. JAMA 289: 76-79.

27. Nouchi, T., Worner, T., Sato, S., and Lieber, C. 1987. Serum procollagen type III N-terminal peptides and laminin P 1 peptide in alcoholic liver disease. Alcohol. Clin. Exp. Res. 11: 287-291.

28. Oddis, C.V. and Finkel, M.S. 1996. NF-kappa B and GTP cyclohydrolase regulate cytokine-induced nitric oxide production by cardiac myocytes. Am. J. Physiol. 270: H1864-1868.

29. Paigen, B., Plump, A., and Rubin, E. 1994. The mouse as a model for human cardiovascular disease and hyperlipidemia. Curr. Opin. Lipidol. 5: 258.

30. Petersen, K.F., Befroy, D., Dufour, S., Dziura, J., Ariyan, C., Rothman, D.L., DiPietro, L., Cline, G.W., and Shulman, G.I. 2003. Mitochondrial dysfunction in the elderly: possible role in insulin resistance. Science 300: 1140-1142.

31. Powell, E.E., Cooksley, W.G., Hanson, R., Searle, J., Halliday, J.W., and Powell, L.W. 1990. The natural history of nonalcoholic steatohepatitis: a follow-up study of fortytwo patients for up to 21 years. Hepatology 11: 74-80.

32. Ratziu, V., Giral, P., Charlotte, F., Bruckert, E., Thibault, V., Theodorou, I., Khalil, L., Turpin, G., Opolon, P., and Poynard, T. 2000. Liver fibrosis in overweight patients. Gastroenterology 118: 1117-1123.

33. Sanyal, A.J. 2002. AGA technical review on nonalcoholic fatty liver disease. Gastroenterology 123: 1705-1725.

34. Shimomura, I., Bashmakov, Y., and Horton, J.D. 1999. Increased levels of nuclear SREBP-1c associated with fatty livers in two mouse models of diabetes mellitus. J. Biol. Chem. 274: 30028-30032.

35. Smith, B.K., Andrews, P.K., and West, D.B. 2000. Macronutrient diet selection in thirteen mouse strains. Am. J. Physiol. Regul. Integr. Comp. Physiol. 278: R797-805.

36. Sone, H. and Kagawa, Y. 2005. Pancreatic beta cell senescence contributes to the pathogenesis of type 2 diabetes in high-fat diet-induced diabetic mice. Diabetologia 48 : 58-67.

37. Springer, T.A. 1994. Traffic signals for lymphocyte recirculation and leukocyte emigration: the multistep paradigm. Cell 76: 301-314.

38. Surwit, R.S., Kuhn, C.M., Cochrane, C., McCubbin, J.A., and Feinglos, M.N. 1988. Diet-induced type II diabetes in C57BL/6J mice. Diabetes 37: 1163-1167.

39. Toye, A.A., Lippiat, J.D., Proks, P., Shimomura, K., Bentley, L., Hugill, A., Mijat, V., Goldsworthy, M., Moir, L., Haynes, A., Quarterman, J., Freeman, H.C., Ashcroft, F.M., and Cox, 
R.D. 2005. A genetic and physiological study of impaired glucose homeostasis control in C57BL/6J mice. Diabetologia 48: 675-686.

40. West, D.B., Boozer, C.N., Moody, D.L., and Atkinson, R.L. 1992. Dietary obesity in nine inbred mouse strains. Am.J. Physiol. 262: R1025-1032.

41. Winzell, M.S. and Ahren, B. 2004. The high-fat diet-fed mouse: a model for studying mechanisms and treatment of impaired glucose tolerance and type 2 diabetes. Diabetes 53: S215-219.

42. Yamakawa, T., Tanaka, S., Yamakawa, Y., Kiuchi, Y., Isoda,
F., Kawamoto, S., Okuda, K., and Sekihara, H. 1995. Augmented production of tumor necrosis factor-alpha in obese mice. Clin. Immunol. Immunopathol. 75: 51-56.

43. Yang, S.Q., Lin, H.Z., Lane, M.D., Clemens, M., and Diehl, A.M. 1997. Obesity increases sensitivity to endotoxin liver injury: implications for the pathogenesis of steatohepatitis. Proc. Natl. Acad. Sci. U.S.A. 94: 2557-2562.

44. Yu, Y.H. and Ginsberg, H.N. 2005. Adipocyte signaling and lipid homeostasis: sequelae of insulin-resistant adipose tissue. Circ. Res. 96: 1042-1052. 